\title{
Blood Pressure Guidelines in Chronic Kidney Disease: A Critical Review
}

\author{
R.C. Thuraisingham M.M. Yaqoob \\ Barts and the London NHS Trust and William Harvey Research Institute, London, UK
}

\section{Key Words}

Guidelines · Standards · Progression of chronic kidney

disease $\cdot$ Cardiovascular diseases

\begin{abstract}
The primary purpose of guidelines should be to improve patient care by providing an avenue for healthcare professionals to participate in the assessment of appropriate care, based on sound medical reasoning and robust scientific knowledge. Guidelines are usually meant to be evidencebased when they are derived from systemic reviews of the relevant literature. Nephrology as a medical subspeciality lags behind other clinical disciplines when it comes to availability of high-quality clinical studies with hard clinical outcomes. In the absence of robust clinical evidence, recommendations in renal guidelines are overwhelmingly opinion-based and reflect the experience of the various experts. Unfortunately, no guidelines are detailed enough to provide recommendations for individual patients with different types and severity of co-morbidities. We propose that guidelines should be viewed as desirables and should not replace a common sense clinical approach to patient care by an autonomously practicing competent clinician.
\end{abstract}

Copyright ๑ 2009 S. Karger AG, Basel

\section{Guidelines}

The literal meaning of guidelines according to the $O x$ ford Dictionary is 'a set of principles directing actions'. Standards which are derived from guidelines are defined as 'a set of principles to which others should conform or be judged'. Unfortunately these dogmatic definitions when applied in clinical practice are viewed by some as biblical statements which must be implemented regardless of the strength of evidence. Increasingly, clinical practice guidelines (CPGs) are coupled with performance measures, which do not take into consideration individual patient's factors or central aspects of patient care. Moreover, coupling performance measures with financial incentives is worrying many healthcare professionals. They resent being judged against collective benchmarks which amounts to intrusion into their professional autonomy and clinical acumen [1].

The primary purpose of CPGs should be to improve patient care by providing an avenue for healthcare professionals to participate in the assessment of appropriate care, based on sound medical reasoning and robust scientific knowledge. CPGs are meant to be evidence-based being derived from systemic reviews of the relevant literature. The evidence is assessed by a panel of experts who then put forward concrete recommendations for patient care. CPGs merely mirror the strength of support-

E-Mailm.m.yaqoob@qmul.ac.uk 
ing evidence. Randomized controlled trials (RCTs), which generate the most robust evidence on treatment efficacy, usually examine only one intervention in a highly selected population with one main health problem. However, in reality, patients usually suffer from at least two or three dominant health problems. Evidence on how to integrate the treatment of several clinical problems in order to optimize health outcomes is negligible. In addition, even RCTs suffer from some inherent weaknesses such as short-term follow-up, small sample size, surrogate endpoints (proteinuria), protocol-driven care which may not reflect typical real-life conditions, strict inclusion criteria such that enrolled patients may not be truly representative of the entire population at risk. Moreover, they are very expensive to conduct and increasingly rely on industrial sponsorships for their implementation and monitoring. Despite usually being a highly selected cohort, patient withdrawal rates in RCTs often exceed 30\% making the implementation of their findings in real-life settings extremely difficult.

Nephrology as a medical subspeciality lags behind other clinical disciplines when it comes to availability of high-quality clinical studies with hard clinical outcomes [2]. The number of RCTs published in nephrology from 1966 to 2002 is much lower than in all other specialties of internal medicine. Some areas of nephrology, in particular glomerulonephritis, the role of different levels of blood pressure (BP) control in progression of chronic kidney disease (CKD), renal outcomes in isolated systolic hypertension, interventions to reduce cardiovascular outcomes in CKD, are clear outliers with either very few or no RCTs to guide clinical decision-making. Furthermore, the quality of RCT reporting in nephrology is relatively poor and has not improved over the past 30 years. This is mainly due to the lack of a double-blinded approach and very frequently failure to perform 'intention-to-treat' analysis.

In the absence of robust clinical evidence, recommendations in renal CPGs are overwhelmingly opinion-based and reflect the experience of various experts. There is growing concern about the influence of the pharmaceutical industry in the form of financial support to clinicians including opinion leaders, medical societies which are involved in the development and promulgating the renal guidelines and disease advocacy groups [3]. Opinionbased recommendations have benefited some industries to the detriment of others. The absence of transparency about these relationships risks undermining the impartiality of the guidelines, which reflects poorly on the renal community in general and threatens our independence.

\section{Blood Pressure Control Recommendations in CKD Guidelines}

\section{A Systolic Blood Pressure (SBP) of $<130$ Is More}

Effective in Slowing the Progression of Diabetic and

Non-Diabetic Kidney Disease with Proteinuria [4-10]

There is a strong evidence to suggest that the prevalence of hypertension ( $>140 / 90 \mathrm{~mm} \mathrm{Hg}$ ) is extremely high in patients with diabetes mellitus with and without diabetic nephropathy and also with various forms of nondiabetic kidney diseases [11-12]. Moreover, a number of prospective studies have shown a strong relationship between higher BP and an increased risk of kidney failure and worsening kidney function in both diabetic and nondiabetic patients [10-12]. Some studies suggest that higher SBP and/or wide pulse pressure (a characteristic of isolated systolic hypertension and reduced arterial wall compliance) is more important for kidney disease progression in both diabetic and non-diabetic kidney disease than diastolic blood pressure (DBP) [10-12].

A SBP goal of $<130 \mathrm{~mm} \mathrm{Hg}$ with the aim of slowing down the rate of decline of renal function has become the holy grail of the management plan of CKD patients. This target was derived from a meta-analysis of 8 trials in diabetic kidney disease and 4 in non-diabetic kidney disease which suggested that a lower BP may slow the progression of kidney disease [13]. Despite the lack of controlling for other factors related to rate of progression in this meta-analysis, a linear relationship between the achieved mean SBP and the mean rate of directly measured or calculated glomerular filtration rate (GFR) decline was seen. However, this linear relationship plateaued below a mean SBP of $<140 \mathrm{~mm} \mathrm{Hg}$, implying that the rate of decline of GFR was similar whether SBP was 140 or $130 \mathrm{~mm} \mathrm{Hg}$ [13].

In type 1 diabetic patients with a mean GFR of $62 \mathrm{ml} /$ $\mathrm{min} / 1.73 \mathrm{~m}^{2}$, a RCT compared intensive [mean arterial pressure (MAP) $<92 \mathrm{~mm} \mathrm{Hg}$ ] to moderate (MAP 100$107 \mathrm{~mm} \mathrm{Hg}$ ) BP control. In this study no significant difference in the rate of progression of kidney failure was seen [14]. Similar results were seen in the hypertensive and normotensive Appropriate Blood Pressure Control in Diabetes (ABCD) studies, which were prospective, randomized, interventional clinical trials with 5 years of follow-up. These studies examined the role of intensive (DBP $<75$ with achieved BP in normotensive of 128/75 and 132/78 in hypertensive patients) versus standard BP control (DBP 80-89 with achieved BP in normotensive of $132 / 81$ and 136/86 in hypertensive patients) in a total of 950 patients with type 2 diabetes mellitus. In both the 
hypertensive and normotensive studies, mean renal function (as assessed by $24 \mathrm{~h}$ creatinine clearance) remained stable during 5 years of either intensive or standard BP intervention in patients with normoalbuminuria $(<30$ $\mathrm{mg} / 24 \mathrm{~h}$ ) or microalbuminuria $(30-300 \mathrm{mg} / 24 \mathrm{~h}$ ) at baseline. By contrast, creatinine clearance in patients with overt diabetic nephropathy ( $>300 \mathrm{mg} / 24 \mathrm{~h}$; albuminuria) at baseline decreased by an average of $5 \mathrm{ml} / \mathrm{min} /$ year in spite of either intensive or standard BP control $[14,15]$. Results of a more recent investigation, the ABCD-2 Valsartan Trial, randomized 129 normotensive patients with type 2 diabetes to receive either placebo or the angiotensin receptor blocker (ARB) valsartan. The BP goals were $<120 / 80 \mathrm{~mm} \mathrm{Hg}$ in the valsartan group and $<140 / 90 \mathrm{~mm}$ $\mathrm{Hg}$ in the placebo group. After a mean of approximately 2 years, those who had received placebo had a mean $( \pm \mathrm{SE}) \mathrm{BP}$ of $124 \pm 11 / 80 \pm 6.5 \mathrm{~mm} \mathrm{Hg}$. The BP of patients in the valsartan group was significantly lower, at $118 \pm 11 / 75 \pm 6 \mathrm{~mm} \mathrm{Hg}(\mathrm{p}<0.001)$. During follow-up, no difference in creatinine clearance between the groups was detected [17].

In non-diabetic patients with CKD the SBP goal of $<130 / 80 \mathrm{~mm} \mathrm{Hg}$ has been investigated in three RCTs [1820]. In the Modification of Diet in Renal Disease (MDRD) study and AASK study (African American Study of Kidney disease and hypertension), patients were randomly assigned to a MAP goal of $<92 \mathrm{~mm} \mathrm{Hg}$ (corresponding to $<125 / 75 \mathrm{~mm} \mathrm{Hg}$ ), compared to a MAP goal of $<107 \mathrm{~mm}$ $\mathrm{Hg}$ (corresponding to $<140 / 90 \mathrm{~mm} \mathrm{Hg}$ ). In the MDRD study a substantial beneficial effect of lower BP was seen only in patients with baseline proteinuria of $>3 \mathrm{~g} /$ day. In patients with moderate renal impairment (creatinine clearance between 25 and $55 \mathrm{ml} / \mathrm{min} / 1.73 \mathrm{~m}^{2}$ ) and proteinuria of between 1 and $3 \mathrm{~g} /$ day, only a modest but statistically significant benefit was seen. In the context of clinical workload it must be added that in the MDRD study only $20 \%$ of the enrolled patients had the characteristics to have benefited from this intervention. However, in the AASK study no beneficial effect of lower BP was observed possibly because the mean proteinuria at baseline in the entire cohort was $<1 \mathrm{~g} /$ day. Recently, investigators of the Ramipril Efficacy In Nephropathy 2 (REIN-2) study were unable to demonstrate an additional benefit for intensified $(<130 / 80 \mathrm{~mm} \mathrm{Hg})$ compared to conventional (DBP $<90 \mathrm{~mm} \mathrm{Hg}$ ) BP control in 338 randomized non-diabetic proteinuric patients with regard to the primary outcome measure of time to end-stage renal disease (ESRD) over 36 months of follow-up. In summary, all these studies have not shown any additional benefit for intensive BP in slowing the progression of renal failure in both diabetic and non-diabetic kidney patients with proteinuria of $<3 \mathrm{~g} /$ day. These observations question the logic of a target SBP of $<130 \mathrm{~mm} \mathrm{Hg}$ in all CKD patients.

\section{Target Blood Pressure for Cardiovascular Disease (CVD) Risk Reduction in CKD Should Be $<130 / 80 \mathrm{~mm} \mathrm{Hg}$}

There is general consensus that patients with CKD carry a very high risk of CVD which is 5-100 times higher than for age- and sex-matched controls in the general population. Several epidemiological studies have demonstrated a graded, independent and highly significant relationship between the level of BP and CVD [11-12]. In the middle-aged and elderly, SBP $>140 \mathrm{~mm} \mathrm{Hg}$ is a more significant CVD risk factor than DBP. Controlled trials in essential hypertension have consistently shown the beneficial effects of lowering BP to $<140 / 90 \mathrm{~mm} \mathrm{Hg}$. There is strong evidence to suggest that in high-risk individuals with diabetes or heart failure a reduction of $\mathrm{BP}$ to even lower values provides added benefits for CVD risk reduction. This has led to the incorporation of a target $\mathrm{BP}$ of $<130 / 80$ for diabetes and congestive heart failure in several guidelines [11-12].

There are hardly any controlled trials regarding BP goals for CVD risk reduction in patients with CKD as primary outcomes. Renal guidelines have used a target $\mathrm{BP}$ goal of $<130 / 80 \mathrm{~mm} \mathrm{Hg}$ as for high-risk non-renal patients in CKD for CVD risk reduction. This is at best an extrapolation of guidelines from the general population on the assumption that types and causes of CVD in CKD patients are similar to non-renal patients. However, there are clear differences in the types and more importantly the causes of both cardiac and vascular disease in patients with CKD and non-renal general populations. Vascular calcification and dysregulated cardiac remodelling are the most dominant cardiovascular problems in CKD patients.

Moreover, both middle-aged and elderly patients with CKD have a very high prevalence of isolated SBP with wide pulse pressure and associated reduced vascular compliance. There is concern that in pursuit of the socalled ideal $\mathrm{BP}$ of $<130 / 80 \mathrm{~mm} \mathrm{Hg}$ by overzealous use of multiple pharmacological agents, doctors may increase the risk of cardiovascular morbidity and mortality by lowering DBP to dangerously low levels. Studies have shown a J-shaped association between DBP levels and risk of adverse cardiac outcomes among patients with and without CKD [21-23]. Interestingly, in the post-hoc analysis of the Irbasartan Diabetic Nephropathy Trial, 
the achieved DBP of $<84$ to $85 \mathrm{~mm} \mathrm{Hg}$ was associated with a higher risk of myocardial infarction and congestive heart failure [21]. Similarly, we recommend the use of clinical acumen and common sense with regard to BP control in patients with postural hypotension, autonomic dysfunction and patients with critical peripheral vascular, cardiac and cerebrovascular disease. The prevalence of these problems is higher in CKD patients and could be exacerbated by BPs of $<130 / 80 \mathrm{~mm} \mathrm{Hg}$ as suggested in the guidelines.

Are Angiotensin-Converting Enzyme (ACE) Inhibitors and/or Angiotensin-II Receptor Blockers (ARBs) Better than Other Antihypertensive Agents in Slowing the

Progression of Kidney Failure or CVD Risk Reduction in CKD Patients?

Most guidelines recommend that ACE inhibitors or ARBs should be first-line antihypertensive therapy in patients with diabetic and non-diabetic nephropathy to reduce proteinuria and retard the progression of renal disease [4-10]. The use of ACE inhibitors or ARBs in diabetes has been included in the new General Medical Services Contract for primary care in the UK, with financial remuneration linked to the prescription of these drugs for this indication [7]. This advice is based on the assumption that inhibition of the renin-angiotensin system with ACE inhibitors or ARBs has specific renoprotective effects independent of their BP-lowering effect [24-26]. Pleiotropic effects of ACE inhibitors on cardiovascular outcomes have also been proposed, based on the results of several large multicentre trials [27-30]. However, in these placebo-controlled trials it was difficult to tease out the BP-independent effects because the use of the active drug reduced BP compared with the control group. However, when ACE inhibitors are compared with an active comparator rather than placebo for cardiovascular effects, no significant advantage of ACE inhibitors has been seen over other classes of BP-lowering drugs [31].

Similarly, the guidelines that advocate using ACE inhibitors and ARBs in renal disease base their recommendations largely on the results of placebo-controlled trials, often using surrogate markers like proteinuria and GFR rather than clinically relevant endpoints [32-34]. However, in a recently published meta-analysis, comparisons of ACE inhibitors or ARBs with other antihypertensive drugs showed no beneficial effect for doubling of creatinine and a very modest benefit for ESRD (relative risk $0.87,0.75-0.99)$. In patients with diabetic nephropathy, no benefit was seen in comparative trials of ACE inhibitors or ARBs on the doubling of creatinine, ESRD, GFR, or creatinine levels. As expected, placebo-controlled trials of ACE inhibitors or ARBs showed greater benefits than comparative trials on all renal outcomes, but were accompanied by substantial reductions in BP in favour of ACE inhibitors or ARBs [35]. However, meta-analysis generated a lot of controversy because the results from the ALLHAT study (Antihypertensive and Lipid Lowering treatment to prevent Heart Attack Trial) [36] were included and were partly responsible for biasing the data against ACE inhibitors. The ALLHAT study, which was one of the largest RCTs in hypertensive patients, reported a posthoc analysis for renal outcomes and lacked detail about proteinuria or serial renal function, but it is extremely unlikely that the investigators missed ESRD, a very robust renal outcome measure. The controversy about ACE inhibitor use in diabetic nephropathy is further fuelled by a recently published Canadian report where the investigators have claimed that ACE inhibitors might actually increase the risk of ESRD in patients with diabetes mellitus treated with this class of antihypertensives for over 10 years [37]. Despite the inherent weakness of any retrospective study such as lack of baseline data about renal function and proteinuria, this study calls for strict monitoring of patients whilst on ACE inhibitors. Similarly, concerns were raised in a recently published series of 100 consecutive $\mathrm{CKD}$ patients in which worsening azotaemia in elderly patients was attributed to ACE inhibitors or ARBs as sustained improvement in estimated GFR often followed the discontinuation of these agents [38]. In the same vein the recent ONTARGET study which ran from 2001 to 2007 and included over 25,000 subjects demonstrated that duel blockade with ACE inhibitors and ARBs in high-risk patients with atherosclerosis or diabetes was associated with worse renal outcomes (dialysis requirement, doubling of creatinine and annual decline of estimated GFR) compared to monotherapy with either ARBs or ACE inhibitors. These adverse renal outcomes were seen despite greater improvement in proteinuria with combination therapy compared to monotherapy [39]. We also have our personal experiences in patients with stable non-progressive CKD developing sudden unexplained deterioration of renal function whilst being on long-term ACE inhibitors with improvement of renal function on withdrawal of the drug. In this regard the short-term follow-up of patients in practically all ACE inhibitor or ARBs trials with use of surrogate outcome measures may have concealed the deleterious effects of these drugs in the later phases of the renal failure. In view of the currently available evidence, treatment decisions for hypertension should be based on the efficacy of BP-lowering 
effect, tolerability and more importantly the cost-benefit ratio of antihypertensive drugs in the long term.

\section{Implementation of Guidelines}

Although up to $70 \%$ of persons with CKD have attained adequate BP control in the setting of clinical trials [40], in trials exploring more ambitious BP targets of $<130 / 80 \mathrm{~mm} \mathrm{Hg}$ only $50 \%$ have achieved the target levels based on the reported mean values in the entire cohort. None of these have reported the proportion of patients consistently achieving target $\mathrm{BP}$ readings. If in an artificial and protocol-driven clinical setting we cannot achieve these targets, then it seems unreasonable to expect $100 \%$ adherence to these targets in day-to-day clinic.

The impact of CKD on the attainment of BP goals and use of antihypertensive therapies has not been thoroughly studied. One study from the National Health and $\mathrm{Nu}$ trition Examination Survey III (1988-1994) found higher BP levels despite greater use of antihypertensive agents among participants with increased serum creatinine levels [41]. In addition, a study from the National Health and Nutrition Examination Survey IV (1999-2002) found that uncontrolled hypertension in persons with CKD appeared almost entirely attributable to high SBP and wide pulse pressure [42]. This observation was extended by a recently published report of a large, multisite, prospective cohort of hypertensive veterans in which the investigators noted that among patients with CKD, greater use of antihypertensives was associated with lower DBP and wide pulse pressure and they cautioned against the pursuit of aggressive control of SBP in order to avoid the potential risk of diastolic hypotension [43]. We support the quotations generated from the debate about the pros and cons of BP guidelines in CKD patients that 'guidelines are for the population and doctor is for an individual patient' and 'one size does not fit all'. Unfortunately no guidelines are detailed enough to provide recommendations for individual patients with different types and severity of comorbidities. We propose that guidelines should be viewed as desirables and should not replace a common sense clinical approach to patient care by autonomous practicing competent clinicians. ticularly GFR and microalbuminuria. As a result of intense publicity, the renal community is under scrutiny and more intense examination. We therefore need much more focused research to convince healthcare professionals that increased attention to renal function and albumin excretion can add significantly to the prevention of cardiovascular and renal disease. We propose more hypothesis-driven studies rather than the current explosion of publications based on the secondary analysis of existing databases using complex statistical techniques. We propose the following questions be addressed urgently by properly conducted observational studies and RCTs with very liberal inclusion criteria:

(1) What do we mean by kidney disease? Do healthy people with GFR values $<60 \mathrm{ml} / \mathrm{min}$ (or even $<90 \mathrm{ml} / \mathrm{min}$ ) in the absence of other renal abnormalities have kidney disease? Do we need to apply the same BP guidelines in these individuals as in patients with known intrinsic renal disease such as diabetes, hypertension or glomerulonephritis?

(2) What are optimum BP goals? There is a real need for studies addressing this issue, particularly for isolated systolic hypertension in elderly patients ( $>60$ years) with both diabetic and non-diabetic kidney disease with the aim of slowing the rate of progression of kidney failure and reducing the risk for CVD in CKD.

(3) How best could clinical trials address the questions of optimum BP targets and most efficacious antihypertensive agents particularly in CKD patients for CVD risk reduction? We feel that the renal community driven by the CKD campaign may be getting ahead of the data. We should now aim to deliver on the promise raised by the publicity drive relating to CKD by addressing the above-mentioned and many more unanswered questions. Failure to deliver robust answers may lead to a loss of confidence in the CKD paradigm with nephrology again being thought of as a niche specialty rather than one concerned with major public health issues [44].

\section{Future Directions}

Renal medicine as a subspeciality has assumed a prominent position with the advent of CKD classification. This has certainly helped in increasing the awareness about the potential epidemic of CKD and with it an epidemic of CVD which could be detected early and even prevented by appropriate measurements of kidney function, par-

References

\footnotetext{
1 Uhlig K, Balk EM, Lau J, Levey AS: Clinical practice guidelines in nephrology - for worse or for better. Nephrol Dial Transplant 2006; 21:1145-1153.

2 Strippoli GF, Craig JC, Schena FP: The number, quality, and coverage of randomized controlled trials in nephrology. J Am Soc Nephrol 2004; 15:411-419.

- 3 Coyne DW: Influence of industry on renal guideline development. Clin J Am Soc Nephrol 2007;2:3-7.
} 
4 Renal Association: Treatment of Adults and Children with Renal Failure: Standards and Audit Measures, ed 3. London, Royal College of Physicians of London and the Renal Association, 2002.

$\checkmark 5$ Williams B, Poulter NR, Brown MJ, et al: Guidelines for management of hypertension: report of the Fourth Working Party of the British Hypertension Society, 2004 - BHS IV. J Hum Hypertens 2004;18:139-185.

6 McIntosh A, Hutchinson A, Marshall S, et al: Clinical guidelines and evidence review for type 2 diabetes. Renal disease: prevention and early management. Sheffield, ScHARR, University of Sheffield, 2002.

7 Royal College of General Practitioners. http://www.rcgp.org.uk/ (accessed March 1, 2005).

$\checkmark 8$ American Diabetes Association: Standards of medical care in diabetes, 2005. Diabetes Care 2005;28(suppl 1):S4-S36.

-9 Chobanian AV, Bakris GL, Black HR, et al: The Seventh Report of the Joint National Committee on Prevention, Detection, Evaluation, and Treatment of High Blood Pressure: The JNC 7 Report. JAMA 2003;289: 2560-2572.

$10 \mathrm{~K} / \mathrm{DOQI}$ clinical practice guidelines on hypertension and antihypertensive agents in chronic kidney disease. Am J Kidney Dis 2004;43(suppl 1):S1-S290.

-11 Chobanian AV, Bakris GL, Black HR, Cushman WC, Green LA, Izzo JL Jr, Jones DW, Materson BJ, Oparil S, Wright JT Jr, Roccella EJ: Seventh Report of the Joint National Committee on Prevention, Detection, Evaluation, and Treatment of High Blood Pressure. Hypertension 2003;42:1206-1252.

12 Palmer BF: Management of hypertension in patients with chronic kidney disease and diabetes mellitus. Am J Med 2008;121(suppl 8): S16-S22.

13 Hart PD, Bakris GL: Managing hypertension in the diabetic patient; in Egan BM, Basile JN, Lackland DT (eds): Hot Topic in Hypertension. Philadelphia, Hanley \& Belfus, 2004, pp 249-252.

-14 Lewis JB, Berl T, Bain RP, Rohde RD, Lewis EJ; the Collaborative Study Group: Effect of intensive blood pressure control on the course of type 1 diabetic nephropathy. Am J Kidney Dis 1999;34:809-817.

$\checkmark 15$ Estacio RO, Jeffers BW, Gifford N, Schrier RW: Effect of blood pressure control on diabetic microvascular complications in patients with hypertension and type 2 diabetes. Diabetes Care 2000;23(suppl 2):SB54-SB64.

- 16 Schrier RW, Estacio RO, Esler A, Mehler P: Effects of aggressive blood pressure control in normotensive type 2 diabetic patients on albuminuria, retinopathy and strokes. Kidney Int 2002;61:1086-1097.

- 17 Estacio RO, Coll JR, Tran ZV, Schrier RW: Effect of intensive blood pressure control with valsartan on urinary albumin excretion in normotensive patients with type 2 diabetes. Am J Hypertens 2006;19:1241-1248.
18 Agodoa LY, Appel L, Bakris GL, et al: Effect of ramipril vs. amlodipine on renal outcomes in hypertensive nephrosclerosis: a randomized controlled trial. JAMA 2001;285:27192728.

19 Klahr S, Levey AS, Beck GJ, Caggiula AW, Hunsicker L, Kusek JW, Striker G; for the Modification of Diet in Renal Disease Study Group: The effects of dietary protein restriction and blood-pressure control on the progression of chronic renal disease. $\mathrm{N}$ Engl J Med 1994;330:877-884.

20 Ruggenenti P, Perna A, Loriga G, Ganeva M, Ene-Iordache B, et al: Blood pressure control for renoprotection in patients with non-diabetic chronic renal disease (REIN-2): multicentre, randomized controlled trial. Lancet 2005;365:939-946.

-21 Berl T, Hunsicker LG, Lewis JB, Pfeffer MA, Porush JG, Rouleau JL, Drury PL, Esmatjes E, Hricik D, Pohl M, Raz I, Vanhille P, Wiegmann TB, Wolfe BM, Locatelli F, Goldhaber SZ, Lewis EJ: Impact of achieved blood pressure on cardiovascular outcomes in the Irbesartan Diabetic Nephropathy Trial. J Am Soc Nephrol 2005; 16:2170-2179.

$>22$ Kovesdy CP, Trivedi BK, Kalantar-Zadeh K, Anderson JE: Association of low blood pressure with increased mortality in patients with moderate to severe chronic kidney disease. Nephrol Dial Transplant 2006;21:12571262.

23 Messerli FH, Mancia G, Conti CR, Hewkin AC, Kupfer S, Champion A, Kolloch R, Benetos A, Pepine CJ: Dogma disputed: can aggressively lowering blood pressure in hypertensive patients with coronary artery disease be dangerous? Ann Intern Med 2006;144: 884-893.

24 Heart Outcomes Prevention Evaluation Study Investigators: Effects of ramipril on cardiovascular and microvascular outcomes in people with diabetes mellitus: results of the HOPE study and MICROHOPE substudy. Lancet 2000;355:253-259.

25 Lewis EJ, Hunsicker LG, Bain RP, Rohde RD the Collaborative Study Group: The effect of angiotensin-converting enzyme inhibition on diabetic nephropathy. N Engl J Med 1993 329:1456-1462.

26 Lewis EJ, Hunsicker LG, Clarke WR, et al; the Collaborative Study Group: Renoprotective effect of the angiotensin-receptor antagonist irbesartan in patients with nephropathy due to type 2 diabetes. N Engl J Med 2001; 345:851-860.

27 EURopean Trial: On reduction of cardiac events with perindopril in stable coronary artery disease investigators: efficacy of perindopril in reduction of cardiovascular events among patients with stable coronary artery disease: randomized, double-blind, placebo-controlled, multicentre trial (the EUROPA study). Lancet 2003;362:782-788.
28 The Heart Outcomes Prevention Evaluation (HOPE) Study Investigators: Effects of an angiotensin-converting enzyme inhibitor, ramipril, on cardiovascular events in highrisk patients. N Engl J Med 2000;342:145153.

29 PROGRESS Collaborative Group: Randomized trial of a perindopril-based blood-pressure-lowering regimen among 6,105 individuals with previous stroke or transient ischaemic attack. Lancet 2001;358:10331041.

30 Weber MA, Julius S, Kjeldsen SE, et al: Blood pressure dependent and independent effects of antihypertensive treatment on clinical events in the VALUE Trial. Lancet 2004;363: 2049-2051.

>31 Turnbull F; Blood Pressure Lowering Treatment Trialists' Collaboration: Effects of different blood-pressure-lowering regimens on major cardiovascular events: results of prospectively-designed overviews of randomized trials. Lancet 2003;362:1527-1535.

32 Jafar TH, Schmid CH, Landa M, et al: Angiotensin-converting enzyme inhibitors and progression of nondiabetic renal disease. A meta-analysis of patient-level data. Ann Intern Med 2001;135:73-87.

33 Giatras I, Lau J, Levey AS; Angiotensin-Converting-Enzyme Inhibition and Progressive Renal Disease Study Group: Effect of angiotensin-converting enzyme inhibitors on the progression of nondiabetic renal disease: a meta-analysis of randomized trials. Ann Intern Med 1997;127:337-345.

-34 Strippoli GF, Craig M, Deeks JJ, Schena FP, Craig JC: Effects of angiotensin-converting enzyme inhibitors and angiotensin II receptor antagonists on mortality and renal outcomes in diabetic nephropathy: systematic review. BMJ 2004;329:828.

- 35 Casas JP, Chua W, Loukgeorgakis S, Vallance $\mathrm{P}$, Smeeth L, Hingorani AD, MacAllister RJ: Effect of inhibitors of the renin-angiotensin system and other antihypertensive drugs on renal outcomes: systematic review and meta-analysis. Lancet 2005;366:20262033.

36 Rahman M, Pressel S, Davis BR, et al: Renal outcomes in high-risk hypertensive patients treated with an angiotensin-converting enzyme inhibitor or a calcium channel blocker versus a diuretic: a report from the Antihypertensive and Lipid-Lowering Treatment to Prevent Heart Attack Trial (ALLHAT). Arch Intern Med 2005;165:936-946.

37 Suissa S, Hutchinson T, Brophy JM, Kezouh A: ACE-inhibitor use and the long-term risk of renal failure in diabetes. Kidney Int 2006; 69:913-919.

38 Onuigbo MA, Onuigbo NT: Late-onset renal failure from angiotensin blockade (LORFFAB) in 100 CKD patients. Int Urol Nephrol 2008;40:233-239. 
-39 Mann JF, Schmieder RE, McQueen M, Dyal L, Schumacher H, Pogue J, Wang X, Maggioni A, Budaj A, Chaithiraphan S, Dickstein K, Keltai M, Metsärinne K, Oto A, Parkhomenko A, Piegas LS, Svendsen TL, Teo KK, Yusuf S; ONTARGET Investigators: Renal outcomes with telmisartan, ramipril, or both, in people at high vascular risk (the ONTARGET study): a multicentre, randomised, double-blind, controlled trial. Lancet 2008;372:547-553.
40 Wright JT Jr, Agodoa L, Contreras G, Greene T, Douglas JG, Lash J, Randall O, Rogers N, Smith MC, Massry S: Successful blood pressure control in the African-American Study of Kidney Disease and Hypertension. Arch Intern Med 2002;162:1636-1643.

41 Coresh J, Wei GL, McQuillan G, Brancati FL, Levey AS, Jones C, Klag MJ: Prevalence of high blood pressure and elevated serum creatinine level in the United States: findings from the third National Health and Nutrition Examination Survey (1988-1994). Arch Intern Med 2001;161:1207-1216.

-42 Peralta CA, Hicks LS, Chertow GM, Ayanian JZ, Vittinghoff E, Feng L, Shlipak MG: Control of hypertension in adults with chronic kidney disease in the United States. Hypertension 2005;45:1-6.
43 Peralta CA, Shlipak MG, Wassel-Fyr C, Bosworth H, Hoffman B, Martins S, Oddone E, Goldstein MK: Association of antihypertensive therapy and diastolic hypotension in chronic kidney disease. Hypertension 2007; 50:474-480.

44 Couser WG: Chronic kidney disease - the promise and the perils. J Am Soc Nephrol 2007; 18:2803-2805. 